\title{
Uma revisão sobre depressão como fator de risco na Doença de Parkinson e seu impacto na cognição
}

\author{
Cláudia Débora Silberman* \\ Jerson Laks** \\ Cláudia Soares Rodrigues*** \\ Eliasz Engelhardt***
}

\section{INTRODUÇÃO}

Demência e depressão são as duas grandes síndromes que podem agravar e trazer conseqüências problemáticas na evolução do processo da Doença de Parkinson (DP). Elas têm influência sobre a qualidade de vida do paciente, aumentam os custos diretos e indiretos do tratamento e sobrecarregam ainda mais o cuidador $^{1-2}$

A depressão ocorre em aproximadamente $40 \%$ dos pacientes com DP ${ }^{3-6}$ com uma incidência de $1,86 \%$ ao ano e um risco cumulativo de $8,6 \%$ ao longo da vida ${ }^{7}$. Porém, há variação na literatura entre $4-70 \%$, incluindo pacientes com depressão maior, depressão menor e distimia ${ }^{2}$. Esta variação ocorre devido aos diferentes cri-

\footnotetext{
* Mestranda em Psiquiatria no Instituto de Psiquiatria da Universidade Federal do Rio de Janeiro.

** Coordenador do Centro de Doença de Alzheimer e outros trantornos mentais na velhice do Instituto de Psiquiatria da Universidade Federal do Rio de Janeiro.

*** Cardiologista, Mestranda em Epidemiologia pelo Núcleo de Estudos de Saúde Coletiva da Universidade Federal do Rio de Janeiro.

**** Coordenador do Setor de Neurologia do Comportamento do Instituto de Neurologia Deolindo Couto da Universidade Federal do Rio de Janeiro.
}

térios diagnósticos ${ }^{2}$ e metodologia utilizadas. A prevalência de depressão na DP é freqüentemente bimodal, ocorrendo em estágios iniciais e finais. Após a fase inicial, os parkinsonianos atravessam uma fase de "lua de mel" com a doença. No entanto, o aumento do comprometimento motor contribui para a recorrência de depressão ${ }^{8}$.

A depressão antecede os sintomas motores em cerca de $25 \%$ dos parkinsonianos deprimidos $^{5}$. Há uma associação positiva entre depressão e subseqüente risco de DP9. Apesar disso, neurologistas não reconhecem ou não diagnosticam depressão e ansiedade em mais de $50 \%$ das vezes em consultas de rotina ${ }^{9}$.

Por outro lado, estudos que avaliaram cognição em transtorno bipolar e unipolar do humor (depressão maior) observam que há disfunção executiva e de memória de trabalho, além de déficit de memória, tanto na fase clínica como no período assintomático ${ }^{10}$. Estes dados apontam para evidência de transtornos cognitivos que seriam fatores de vulnerabilidade para depressão, com impacto sobre a evolução da doença.

O tratamento precoce e eficaz da depres- 
são tem impacto positivo sobre o desempenho cognitivo dos parkinsonianos ${ }^{11,3}$, o que chama a atenção para a necessidade eventual de reconhecimento da sintomatologia depressiva para a melhora destes pacientes. Além disso, o diagnóstico precoce de depressão pode dirimir dúvidas sobre a ocorrência de demência na evolução da DP, uma vez que este diagnóstico diferencial nem sempre é simples.

A busca por consenso para este assunto leva à indagação sobre qual conjunto de artigos e pesquisas podem nortear a prática clínica. Neste sentido, a revisão de artigos de estudos prospectivos, seccionais e de caso controle sobre depressão, cognição e DP pode ser útil para a compreensão do tema.

Objetivos: Esta revisão tem dois objetivos. 1. Examinar o impacto da depressão sobre a cognição na DP. 2. Examinar o papel da depressão como fator de risco tanto para DP como para transtorno cognitivo na DP.

Metodologia: Revisão na literatura internacional, Medline, de artigos avaliando a função cognitiva de parkinsonianos com e sem depressão no período de 1967 até 2003.

Foram incluídos na revisão somente artigos de pesquisas clínicas seccionais, prospectivas ou de caso controle. Os relatos de casos, os artigos de revisão, de neurocirurgia, neuroimagem e psicofarmacologia foram excluídos deste trabalho por não interessarem diretamente ao objetivo traçado. Os artigos que tratavam exclusivamente de depressão na DP ou de cognição na DP foram excluídos.

\section{RESULTADOS}

A tabela 1 mostra artigos sobre o impacto da depressão na cognição de parkinsonianos, e a tabela 2 apresenta artigos que associam ou não a depressão como fator de risco para DP e para transtorno cognitivo em parkinsonianos.

\section{Impacto da depressão sobre a cognição na $D P$}

Os estudos abordando o impacto da depressão sobre a cognição na DP (tabela 1) contaram com um total de 971 pacientes e 213 controles saudáveis.

Dois artigos ${ }^{11-12}$ observaram que parkinsonianos com depressão maior apresentam pior desempenho cognitivo em testagens neuropsicológicas, especialmente em funções frontais, quando comparados com parkinsonianos sem depressão. Mayeux ${ }^{13}$ encontrou resultado semelhante. No entanto, correlacionou este comprometimento cognitivo à gravidade da depressão (especialmente para cálculo, digit span e habilidades visuomotoras). Um outro trabalho ${ }^{14}$ afirma que a DP afeta todos os níveis de funcionamento cognitivo e atribui à depressão a causa de hipomnesia na DP. No entanto, Younjohn e cols ${ }^{15}$ concluiu que a depressão não é responsável por todas as alterações neuropsicológicas associadas com a DP.

Há associação positiva entre apatia e déficit cognitivo em parkinsonianos ${ }^{16}$. As dificuldades enfrentadas na clínica são compatíveis com os limites tênues entre as três síndromes supracitadas.

Alterações quantitativas, mas não qualitativas dos déficits cognitivos são descritas em pacientes com depressão e DP ${ }^{17}$. Dois artigos não encontraram qualquer impacto da depressão na cognição de parkinsonianos ${ }^{18-19}$, sendo que um deles ${ }^{19}$ ainda descreve melhor desempenho dos pacientes com depressão em testes frontais.

Depressão como fator de risco tanto para $D P$ como para transtorno cognitivo na DP

Um artigo ${ }^{9}$ encontrou associação fortemente positiva entre depressão e subseqüente incidência de DP (tabela 2). Há evidências também de que a depressão na DP seja conseqüência de disfunção cerebral ${ }^{1}$. Parkinsonianos com o início da sintomatologia da DP pelo dimídio direito ${ }^{20}$ e cujo hemisfério dominante está afetado primariamente apresentam depressão mais grave. Estes pacientes têm história familiar para depressão, o que serviria como um marcador biológico.

Parkinsonianos com depressão maior apresentam declínio cognitivo significativamente maior, deterioração em atividades de vida diária e maior avanço em estágios da escala de Hoehn e Yahr do que DP com depressão menor ou sem depressão ${ }^{21}$.

A depressão em parkinsonianos está associada com o avanço da gravidade da DP, com o estágio avançado de Hoehn e $\mathrm{Yahr}^{22}$ e alta pontuação na Unified Parkinson's Disease Rating Scale (UPDRS) ${ }^{23}$. Do ponto de vista da cognição e evolução da doença, pode-se dizer que a evolução é mais rápida, com ocorrência de quedas, baixa pontuação no Mini Exame do Estado Mental (MEEM) ${ }^{24}$ e na Escala Schawab e England ${ }^{25}$ e déficit cognitivo. Quanto aos aspectos motores da doença, há mais bradicinesia axial, alterações na marcha e no balanço; 
Tabela 1. Artigos sobre o impacto da depressão sobre a cognição na DP.

\begin{tabular}{|c|c|c|c|c|}
\hline ARTIGO & AUTOR & $\mathrm{N}$ & ANO & RESULTADOS \\
\hline $\begin{array}{l}\text { De pression, intellectual } \\
\text { impairment and } \\
\text { Parkinson disease }\end{array}$ & Mayeux $\mathrm{R}$, et al. ${ }^{13}$ & $\begin{array}{l}55 \text { com DP e demência } \\
\text { e } 31 \text { das esposas. }\end{array}$ & 1981 & $\begin{array}{l}\text { Existe correlação entre gravidade da } \\
\text { depressão e comprometimento } \\
\text { cognitivo, especialmente para cálculo, } \\
\text { digit span e habilidades visuomotoras. }\end{array}$ \\
\hline $\begin{array}{l}\text { Depression and Cognitive } \\
\text { Impairment in Parkinson } \\
\text { Disease }\end{array}$ & Starkstein S E, et al. ${ }^{12}$ & $\begin{array}{l}105 \text { com DP (15 } \\
\text { parkinsonianos } \\
\text { com depressão } \\
\text { maior e } 19 \text { com } \\
\text { depressão menor). }\end{array}$ & 1989 & $\begin{array}{l}\text { Parkinsonianos com depressão maior, } \\
\text { quando comparados a parkinsonianos sem } \\
\text { depressão, apresentam pior desempenho } \\
\text { cognitivo em testagens neuropsicológicas, } \\
\text { especialmente em funções frontais. }\end{array}$ \\
\hline $\begin{array}{l}\text { Neuropsycological } \\
\text { Impairment, Depression, } \\
\text { and Parkinson's Disease }\end{array}$ & Younjohn J, et al. ${ }^{15}$ & $\begin{array}{l}89 \text { parkinsonianos } \\
\text { sem demência, } \\
19 \text { parkinsonianos com } \\
\text { demência e } 64 \text { controles. }\end{array}$ & 1992 & $\begin{array}{l}\text { A depressão não é responsável por todas } \\
\text { as alterações neuropsicológicas } \\
\text { associadas com a DP. }\end{array}$ \\
\hline $\begin{array}{l}\text { Neuropsychological } \\
\text { Impairment in Parkinson's } \\
\text { Disease With and Without } \\
\text { Depression. }\end{array}$ & Troster Al, et al. ${ }^{17}$ & $\begin{array}{l}44 \text { parkinsonianos sem } \\
\text { depressão, } 44 \\
\text { parkinsonianos com } \\
\text { depressão e } 44 \text { controles } \\
\text { normais. }\end{array}$ & 1995 & $\begin{array}{l}\text { A depressão influencia na quantidade e } \\
\text { não na qualidade dos déficits cognitivos } \\
\text { associados á DP. }\end{array}$ \\
\hline $\begin{array}{l}\text { Cognitive Functions in } \\
\text { Major Depression and } \\
\text { Parkinson Disease }\end{array}$ & Kuzis G, & $\begin{array}{l}19 \text { com DP e depressão } \\
\text { maior; } 31 \text { com DP sem } \\
\text { depressão, } 27 \text { com } \\
\text { depressão sem DP e } 12 \\
\text { controles saudáveis. }\end{array}$ & 1997 & $\begin{array}{l}\text { Parkinsonianos com depressão maior } \\
\text { apresentam déficits cognitivos que não } \\
\text { podem ser completamente explicados pela } \\
\text { depressão maior. Déficits cognitivos } \\
\text { frontais podem resultar da interação entre } \\
\text { fatores neuropatológicos da DP e o } \\
\text { mecanismo da depressão maior. }\end{array}$ \\
\hline $\begin{array}{l}\text { Depression and } \\
\text { Parkinson's Disease: a } \\
\text { study of a series of } 135 \\
\text { Parkinson's patients. }\end{array}$ & Anguenot $\mathrm{A}$, et al. ${ }^{19}$ & $\begin{array}{l}135 \text { com DP (mais da } \\
\text { metade apresentavam } \\
\text { depressão). }\end{array}$ & 2002 & $\begin{array}{l}\text { Os pacientes deprimidos não } \\
\text { apresentaram diminuição significativa no } \\
\text { desempenho cognitivo e apresentaram } \\
\text { escores mais elevados em testes frontais. }\end{array}$ \\
\hline $\begin{array}{l}\text { Effects of depression and } \\
\text { Parkinson's disease on } \\
\text { cognitive functioning }\end{array}$ & Norman S, et al. ${ }^{14}$ & $\begin{array}{l}19 \text { com DP e depressão, } \\
19 \text { com DP sem } \\
\text { depressão, } 19 \text { com } \\
\text { depressão e } 10 \text { controles } \\
\text { normais (total de } 76 \text { ). }\end{array}$ & 2002 & $\begin{array}{l}\text { A DP afeta todos os níveis de } \\
\text { funcionamento cognitivo e a diminuição } \\
\text { da memória na DP está primariamente } \\
\text { relacionada á depressão. }\end{array}$ \\
\hline Apathy in PD & Pluck GC, et al. ${ }^{16}$ & $\begin{array}{l}45 \text { parkinsonianos, } 17 \\
\text { pacientes com } \\
\text { osteoartrite. }\end{array}$ & 2002 & $\begin{array}{l}\text { Apatia na DP está associada á déficit } \\
\text { cognitivo e pode ser distinguida de outros } \\
\text { sintomas psiquiátricos. }\end{array}$ \\
\hline $\begin{array}{l}\text { The Prevalence and } \\
\text { Correlates of } \\
\text { Neuropsychiatric } \\
\text { Symptoms in a } \\
\text { Population With } \\
\text { Parkinson's Diseas } \\
\text { in Mexico }\end{array}$ & Ringman JM, et al. ${ }^{18}$ & $\begin{array}{l}40 \text { com DP e } 83 \\
\text { controles. }\end{array}$ & 2002 & $\begin{array}{l}\text { Não foi encontrada relação entre a } \\
\text { duração da doença, gravidade, déficit } \\
\text { cognitivo e sintomas neuropsiquiátricos. }\end{array}$ \\
\hline
\end{tabular}


Tabela 2. Depressão como fator de risco para DP e para transtorno cognitivo na DP.

\begin{tabular}{|c|c|c|c|c|}
\hline$\overline{\text { ARTIGO }}$ & AUTORES & $\mathrm{N}$ & ANO & RESULTADOS \\
\hline $\begin{array}{l}\text { A prospective } \\
\text { longitudinal study of } \\
\text { depression, cognitive } \\
\text { decline, and physical } \\
\text { impairments in patients } \\
\text { with Parkinson's disease. }\end{array}$ & Starkstein SE, et al. ${ }^{21}$ & $\begin{array}{l}105 \text { com DP. } \\
92 \text { receberam a } \\
\text { mesma avaliação } \\
12 \text { meses depois. }\end{array}$ & 1992 & $\begin{array}{l}\text { Pacientes com depressão maior } \\
\text { apresentaram declínio cognitivo } \\
\text { significativamente maior, deterioração em } \\
\text { atividades de vida diária e maior avanço } \\
\text { em estágios da escala de Hoehn e Yahr do } \\
\text { que os pacientes com depressão menor ou } \\
\text { sem depressão. }\end{array}$ \\
\hline $\begin{array}{l}\text { Risk Factor for } \\
\text { depression in } \\
\text { Parkinson disease } \\
\end{array}$ & Tandberg $\mathrm{E}$, et al. ${ }^{27}$ & 245 com DP. & 1997 & $\begin{array}{l}\text { Déficit cognitivo e presença de alteração } \\
\text { do pensamento são preditores } \\
\text { significativos de depressão maior na DP. }\end{array}$ \\
\hline $\begin{array}{l}\text { Depressive symptoms } \\
\text { in patients with PD. }\end{array}$ & Popovska A, et al. ${ }^{30}$ & 62 com DP. & 1997 & $\begin{array}{l}\text { Pacientes com DP, idade mais avançada e } \\
\text { do sexo feminino apresentam com maior } \\
\text { frequiência depressão. }\end{array}$ \\
\hline $\begin{array}{l}\text { The correlation of } \\
\text { depression with } \\
\text { functional activity in } \\
\text { Parkinson's disease }\end{array}$ & Liu CY, et al. ${ }^{40}$ & $\begin{array}{l}18 \text { com DP e depressão } \\
\text { maior, } 25 \text { com DP e } \\
\text { outros transtornos } \\
\text { depressivos e } 58 \text { com } \\
\text { DP e sem depressão. }\end{array}$ & 1997 & $\begin{array}{l}\text { Parkinsonianos apresentam elevada } \\
\text { freqüência de depressão. Significativa } \\
\text { correlação entre depressão e atividades } \\
\text { de vida diária foi encontrada. }\end{array}$ \\
\hline $\begin{array}{l}\text { Depression in PD is not } \\
\text { Accompanied by More } \\
\text { Corticotrophin - } \\
\text { Releasing Hormone } \\
\text { Expressing Neurons in } \\
\text { the Hypotalamic } \\
\text { Paraventricular Nucleus. }\end{array}$ & $\begin{array}{l}\text { Hoogendijk WJG, } \\
\text { et al. }{ }^{35}\end{array}$ & $\begin{array}{l}6 \text { com DP e depressão, } \\
6 \text { com DP e sem } \\
\text { depressão e } 6 \text { controles }\end{array}$ & 1997 & $\begin{array}{l}\text { Ativação de neurônios que liberam } \\
\text { corticotropina no núcleo paraventricular, } \\
\text { recentemente observados na depressão } \\
\text { diopática, não acompanham a depressão } \\
\text { em parkinsonianos. }\end{array}$ \\
\hline $\begin{array}{l}\text { Depression in classic } \\
\text { versus akinetic-rigid } \\
\text { Parkinson' disease } \\
\end{array}$ & Starkstein SE, et al. ${ }^{31}$ & $\begin{array}{l}78 \text { com DP tremulante } \\
\text { e } 34 \text { com DP } \\
\text { rígido-acinético }\end{array}$ & 1998 & $\begin{array}{l}\text { Associação significativa entre depressão } \\
\text { maior e DP rígido-acinético. }\end{array}$ \\
\hline Depression and PD & Errea JM, et al. ${ }^{29}$ & $\begin{array}{l}62 \text { com DP e depressão. } \\
48 \text { com DP. }\end{array}$ & 1999 & $\begin{array}{l}\text { A depressão está associada com a } \\
\text { duração da DP. Uma relação inversa entre } \\
\text { depressão e o funcionamento cognitivo } \\
\text { foi encontrada nos parkinsonianos. }\end{array}$ \\
\hline $\begin{array}{l}\text { Parkinson' disease, side } \\
\text { affected and depression }\end{array}$ & Serrano-Dueñas M. ${ }^{41}$ & $\begin{array}{l}63 \text { com DP nos estágios } \\
1 \text { ou 1,5 (Hoehn e Yahr). }\end{array}$ & 2000 & $\begin{array}{l}\text { Parkinsonianos cujo lado dominante está } \\
\text { afetado primariamente apresentam } \\
\text { depressão mais grave. }\end{array}$ \\
\hline $\begin{array}{l}\text { Cognitive and motor } \\
\text { function in patients } \\
\text { with PD with and } \\
\text { without depression. }\end{array}$ & Cubo $E$, et al. ${ }^{28}$ & 88 com DP. & 2000 & $\begin{array}{l}\text { Baixo escore no MEEM, bradicinesia axial, } \\
\text { alterações na marcha e no balanço são } \\
\text { preditores significativos de depressão } \\
\text { na DP. }\end{array}$ \\
\hline $\begin{array}{l}\text { The effects of long-term } \\
\text { therapy on depression } \\
\text { level in the novo patients } \\
\text { with PD. }\end{array}$ & Choi C, et al. ${ }^{32}$ & $\begin{array}{l}34 \text { com DP e } \\
70 \text { saudáveis }\end{array}$ & 2000 & $\begin{array}{l}\text { O tratamento com Levodopa não altera a } \\
\text { depressão na DP. Existem evidências que } \\
\text { sugerem o papel de mecanismos não } \\
\text { dopaminérgicos na depressão de } \\
\text { parkinsonianos. }\end{array}$ \\
\hline $\begin{array}{l}\text { Non-recognition of } \\
\text { depression and other } \\
\text { non-motor symptoms } \\
\text { in PD. }\end{array}$ & Shulman LM, et al..$^{38}$ & 101 com DP. & 2001 & $\begin{array}{l}\text { Durante visitas de rotina, neurologistas não } \\
\text { identificaram depressão, ansiedade e } \\
\text { fadiga em mais da metade dos } \\
\text { parkinsonianos. }\end{array}$ \\
\hline
\end{tabular}




\begin{tabular}{|c|c|c|c|c|}
\hline ARTIGO & AUTORES & $\mathrm{N}$ & ANO & RESULTADOS \\
\hline $\begin{array}{l}\text { What contributes to } \\
\text { depression in Parkinson' } \\
\text { disease? }\end{array}$ & Scharg A, et al. ${ }^{26}$ & 97 com DP. & 2001 & $\begin{array}{l}\text { Depressão em parkinsonianos está } \\
\text { associada com o avanço da gravidade da } \\
\text { DP, recente deterioração da doença e } \\
\text { ocorrência de quedas. }\end{array}$ \\
\hline $\begin{array}{l}\text { Markers for depression } \\
\text { in PD }\end{array}$ & Leentjens AFG, et I. ${ }^{20}$ & $\begin{array}{l}121 \text { com DP e } 40 \\
\text { com DP } \\
\text { e depressão. }\end{array}$ & 2002 & $\begin{array}{l}\text { História familiar de depressão foi o } \\
\text { marcador mais importante. O início da DP } \\
\text { no lado direito foi o único marcador, } \\
\text { específico da doença, que melhorou o } \\
\text { modelo estudado. }\end{array}$ \\
\hline $\begin{array}{l}\text { Major depressive } \\
\text { disorder in Parkinson's } \\
\text { disease: a register - } \\
\text { based study. }\end{array}$ & Nilsson FM, et al. ${ }^{1}$ & $\begin{array}{l}211245 \text { pacientes } \\
\text { com osteoartrite, } \\
\text { diabetes e DP. }\end{array}$ & 2002 & $\begin{array}{l}\text { Depressão em pacientes com DP é } \\
\text { conseqüência de disfunção cerebral. } \\
\text { Mulheres apresentam maior risco de } \\
\text { depressão na DP. }\end{array}$ \\
\hline $\begin{array}{l}\text { Increased risk of } \\
\text { Parkinson's disease } \\
\text { after depression. } \\
\text { A retrospective } \\
\text { cohort study }\end{array}$ & $\begin{array}{l}\text { Schuurman AG, } \\
\text { et al. }{ }^{9}\end{array}$ & $\begin{array}{l}\text { 1,358 com depressão e } \\
67,570 \text { sem depressão. }\end{array}$ & 2002 & $\begin{array}{l}\text { Associação fortemente positiva foi } \\
\text { encontrada entre depressão e } \\
\text { subseqüente incidência de DP. }\end{array}$ \\
\hline $\begin{array}{l}\text { Depression in PD: } \\
\text { clinical correlates } \\
\text { and outcome }\end{array}$ & Rojo A, et al. ${ }^{25}$ & $\begin{array}{l}353 \text { - avaliação única e } \\
184 \text { - estudo prospectivo. }\end{array}$ & 2003 & $\begin{array}{l}\text { Sexo feminino, estágio elevado de Hoehn } \\
\text { e Yahr, escore elevado de UPDRS, baixos } \\
\text { escores no MEEM e na Escala Schawab e } \\
\text { England estão associados a sintomas } \\
\text { expressivos. }\end{array}$ \\
\hline
\end{tabular}

idade mais avançada, sexo feminino e presença de alteração do pensamento são outros fatores de risco para depressão na $\mathrm{DP}^{21,26-29,25,30}$.

\section{DISCUSSÃO}

A existência de diferenças entre a depressão da DP e transtornos do humor, o impacto da depressão na cognição de parkinsonianos, o substrato neuroanatômico da depressão na DP e os fatores de risco para depressão na DP ainda são assuntos controversos.

\section{Impacto da depressão na cognição de parkinsonianos}

Os trabalhos sobre o impacto da depressão na cognição de parkinsonianos apresentam afirmações divergentes: parkinsonianos com depressão maior apresentam déficits cognitivos especialmente em funções frontais ${ }^{11,31}$ e a depressão influencia na quantidade e não na qualidade dos déficits cognitivos de parkinsonianos ${ }^{17}$. Em relação aos testes neuropsicológicos, os parkinsonianos apresentam escores mais elevados em testes frontais, apesar de apresentarem diminuição significativa do desempenho $\operatorname{cog}$ itivo $^{19}$, mas não parece haver relação entre sintomas neuropsiquiátricos e déficit cognitivo ${ }^{18}$. A depressão não é responsável por todas as alterações neuropsicológicas associadas com a $\mathrm{DP}^{15,11}$ e existe correlação entre gravidade da depressão e comprometimento cognitivo ${ }^{13}$.

\section{Possíveis causas da alta incidência de depressão na $D P$}

Uma das questões que permanece sem resposta é a causa da alta incidência de depressão na DP. Cogitam-se duas causas: psicológica (em conseqüência das questões relacionadas com um doença de conseqüências motoras limitantes e até mesmo incapacitantes) e/ou disfunção cerebral. Estudos que definem a depressão em parkinsonianos como conseqüência de disfunção cerebral ${ }^{1}$ são corroborados por trabalhos que evidenciam associação significativa entre depressão maior e DP rígidoacinético ${ }^{12}$ e envolvimento de mecanismos não dopaminérgicos na depressão de parkinsonianos $\mathrm{DP}^{32}$. A vulnerabilidade biológica da depressão em parkinsonianos é ainda confirmada por um estudo que encontrou associação fortemente positiva entre depressão e subseqüente incidência de DP $^{9}$. Em cerca de $25 \%$ dos par- 
kinsonianos deprimidos, a depressão antecede os sintomas motores da DP5. Cogita-se a "hipótese serotonérgica" como possível explicação para o aumento da incidência de DP em pacientes com transtornos depressivos anteriores. Essa hipótese considera a baixa atividade serotonérgica em cérebros de pacientes com DP como fator de risco para depressão ${ }^{2,33-34}$. A serotonina tem função inibitória da liberação de dopamina no striatum. Assim, a redução da atividade de serotonina pode ser um mecanismo compensatório para a redução da atividade de dopamina na DP. Por outro lado, a redução da atividade de serotonina aumenta o risco de depressão. Logo, os parkinsonianos teriam uma vulnerabilidade biológica para transtornos depressivos. Como a redução da serotonina já existe antes do início dos sintomas motores, o risco de depressão também aumenta antes de sintomas parkinsonianos serem aparentes. Existe a possibilidade do aumento da incidência de DP em pacientes com uma história prévia de depressão ser o reflexo da presença desse fator de risco biológico para depressão em estágios da DP pré-clínicos ${ }^{9}$. Logo, a interação entre depressão e DP é complexa e bidirecional. Pode-se afirmar que depressão é um fator de risco para DP, assim como DP é um fator de risco para depressão.

A depressão em parkinsonianos apresenta evidências que sugerem o papel de mecanismos não dopaminérgicos ${ }^{35}$ e há indícios de que a depressão idiopática difere da depressão em parkinsonianos. A ativação de neurônios que liberam corticotropina no núcleo paraventricular, recentemente observados na depressão idiopática, não ocorre na depressão em parkinsonianos ${ }^{36}$.

\section{Depressão como fator de risco para transtornos cognitivos na DP}

Ao se avaliar a depressão como fator de risco para transtornos cognitivos em parkinsonianos, observa-se que os dados sobre a prevalência de depressão e déficit cognitivo na DP são inconclusivos, com grande margem percentual entre os autores. As amostras estudadas não permitem qualquer afirmação sobre o assunto, uma vez que não se conhece a prevalência de déficits cognitivos em parkinsonianos com e sem depressão. Torna-se, assim, impossível calcular o tamanho amostral ideal para a realização das pesquisas selecionadas. Mais importante do que o tamanho amostral seria a discussão da validade de cada amostra. No entanto, os estudos geralmente utilizam amos- tras não-aleatórias e não discutem a validade dessas amostras.

A heterogeneidade da nomenclatura utilizada também afeta os resultados dos estudos. Alguns estudos utilizaram o diagnóstico de depressão maior, distimia e depressão menor de acordo com a DSM-III-R 37,11-12,19. Observam-se ainda quatro artigos que aplicaram escalas para a realização do diagnóstico de depressão, sem a utilização de critérios clínicos estruturados como na DSM-IV38,13,17-18, nos quais o diagnóstico de depressão foi realizado através do Inventário de Beck (IDB). O IDB a escala Hospital Anxiety and Depression Scale (HADS) foram utilizados para investigar o humor de parkinsonianos e realizar o diagnóstico de depressão ${ }^{16}$.

\section{Diagnóstico da síndrome depressiva na $D P$}

O diagnóstico diferencial entre as síndromes depressiva, apato-abúlica e demencial na DP pode se constituir em difícil tarefa. Na DP sintomas como bradicinesia, fadiga, dificuldade de concentração, alterações do sono e diminuição da libido podem estar presentes, sem que o paciente esteja deprimido ${ }^{16}$. Eventualmente 0 parkinsoniano pode descontinuar atividades sociais em virtude de discinesias incapacitantes ou mesmo desconforto com sua aparência que pode mimetizar a de um paciente deprimido (bradicinesia e diminuição da expressão facial).

Neurologistas custam a reconhecer depressão na vigência de $\mathrm{DP}^{39}$ durante visitas de rotina. Os médicos falharam em identificar depressão, ansiedade e fadiga em mais da metade dos parkinsonianos ${ }^{40}$. A depressão aumenta o risco de declínio cognitivo ou demência na $\mathrm{DP}^{41}$. Assim, reconhecer depressão o mais precocemente possível e tratá-la pode ser importante para alteração do curso clínico da doença.

Algumas críticas devem ser levadas em consideração ao se avaliar os resultados gerados pela presente revisão. Somente um dos autores avaliou toda a base de dados (CDS), o que pode ter deixado de fora alguns artigos que eventualmente preenchessem critérios de inclusão para a revisão. Outro ponto passível de crítica é o fato desta revisão ter se limitado à base Medline, em Inglês. Como decorrência, dados positivos e negativos, com possível relevância clínica para o assunto, podem ter sido negligenciados devido a isto. A revisão realizada apresenta, possivelmente, um viés de publicação, pois raramente os artigos publicados evidenciam associações negativas ou resulta- 
dos sem significância estatística. De todos os artigos selecionados, apenas três constituem uma exceção, dois sobre fisiopatologia da depressão na DP e um sobre o impacto da depressão sobre a cognição nos pacientes com $\mathrm{DP}^{32,19,35}$

\section{CONCLUSÃO}

A revisão deste trabalho não permite concluir que a depressão tenha impacto sobre a cognição nos pacientes com DP. Cinco artigos concluem afirmando esta hipótese e quatro não a confirmam.

Pode-se afirmar que a interação entre depressão e DP é complexa e bidirecional, ou seja: depressão é um fator de risco para DP, assim como DP é um fator de risco para depressão. É possível se traçar um perfil mais homogêneo do paciente deprimido com DP que evolui com transtorno cognitivo.

Divergências e contradições marcam o conjunto de estudos sobre cognição, DP e depressão. Há limites tênues entre os déficits cognitivos relacionados à depressão e à própria DP. O entendimento do substrato neuroanatômico da depressão em parkinsonianos pode contribuir para o conhecimento de tais limites. Assim, não foi possível definir a depressão como um fator de risco para transtornos cognitivos na DP. Estudos que utilizem critérios diagnósticos definidos e com amostras representativas da população podem trazer esclarecimento sobre o assunto.

\section{REFERÊNCIAS BIBLIOGRÁFICAS}

1. Nilsson F, Kessing L, Sorensen T, Andersen PK, Bolwing T. Major depressive disorder in Parkinson's disease: a register- based study. Acta Psychiatr Scand 2002; 106:202-11

2. Rabinstein LM, Shulman LM. Management of behavioral and psychiatric problems in Parkinson's disease. Parkinsonism Relat Disord 2001; 7:41-50.

3. Starkstein SE, Preziosi TJ, Bolduc PL, Robinson RG. Depression in Parkinson's disease. J Nerv Ment Dis 1990 178:27-31

4. Hughes AJ, Daniel SE, Kilford L, Lees AJ. Accuracy of clinical diagnosis of idiopathic Parkinson's disease: a clinic-pathological study of 100 cases. J Neurol Neurosurg Psychiatry 1992; 55:181-4.

5. Lieberman A. Managing the neuropsychiatric symptoms of Parkinson's disease. Neurology 1998; 50:33-8.

6. Colcher A, Simuni T. Clinical manifestations of Parkinson's disease. Med Clin North Am 1999; 83:32747.

7. Dooneief G, Mirabello E, Bell K, Marder K, Stern Y, Mayeux R. An estimate of the incidence of depression in idiopathic Parkinson's disease. Arch Neurol 1992; 49:305-7.
8. Brooks D, Doder M. Depression in Parkinson's disease. Curr Opin Neurol 2001; 14:465-70.

9. Schuurmann AG, Akker M, Ensinck KTJL, Metsemakers JFM, Knottnerus JA, Leentjens AFG, et al. Increased risk of Parkinson's disease after depression. A retrospective cohort study. Neurology 2002; 58:1501-4.

10. Laks J, Marinho V, Rozenthal M, Engelhardt E. Neuropsicologia da depressão. Rev bras Neurol 1999; 35:97-102.

11. Kuzis G, Sabe L, Tiberti, C, Leiguarda R, Starkstein, S. Cognitive Functions in Major Depression and Parkinson Disease. Arch Neurol 1997; 54:982-6.

12. Starkstein SE, Petracca G, Chemerinski E, Tesón A, Sabe L, Merello M, et al. Depression in classic versus akinetic-rigid Parkinson's disease. Mov Disord , 1998; 13:29-33.

13. Mayeux R, Stern Y, Rosen, J, Leventhal, J. Depression, intellectual impairment and Parkinson's disease. Neurology $1981 ; 31: 645-50$.

14. Norman S, Troster AI, Fields JA, Brooks R. Effects of depression and Parkinson's disease on cognitive functioning. J Neuropsychiatry Clin Neurosc 2002; 12:31-6.

15. Youngjohn JR, Beck J, Jogerst G, Caine C. Neuropsychological Impairment, Depression and Parkinson's disease. Neuropsychology 1992; 6:149-58.

16. Pluck GC, Brown RG. Apathy in Parkinson's disease. J Neurosurg Psychiatry 2002; 73:636-42.

17. Troster A, Stalp L, Paolo A, Fields J, William K. Neuropsychological Impairment in Parkinson's Disease With and Without Depression. Arch Neurol 1995; 52:1164-9.

18. Ringman JM, Diaz-Olavarrieta C, Rodriguez Y. The Prevalence and Correlates of europsychiatric Symptoms in a Population With Parkinson's Disease in Mexico. Neuropsychiatr Neuropsychol Behav Neurol 2002; 15: 99-105.

19. Anguenot A, Loll PY, Neau JP, Ingrand P, Gil R. Depression and Parkinson's disease: study of series of 135 Parkinson's 1 patients. Can J Neurol Sci 2002; 29:139-46.

20. Leentjens AFG, Lousberg R, Verhey FRJ. Markers for depression in Parkinson's disease. Acta Psychiatric Scand 2002; 106:196-201.

21. Starkstein SE, Mayberg HS, Leiguarda R, Preziosi TJ, Robinson RG. A prospective longitudinal study of depression, cognitive decline, and physical impairments in patients with Parkinson's disease. J Neurol Neurosurg Psychiatry 1992; 55:377-82.

22. Hoehn MM, Yahr MD. Parkinsonism: onset, progression, and mortality. Neurology 1967; 17:427-42.

23. Fahn S, Elton E. The UPDRS Development Committee. Unified Parkinson' Disease Rating Scale. In: Fahn S, Marsden CD, Goldstein M, Calne CD, eds. Recent Developments in Parkinson's Disease. New York: Macmillan Publishing CO Inc. 1987: 153-63.

24. Folstein ME, Folstein SE e McHugh PR. Mini Mental State. A Practical Method for Grading the Cognitive State of Patients for the Clinician. J Psychiatr Res 1975; 12: 189-98.

25. Rojo A, Aguilar M, Garolera MT, Cubo E, Navas I, Quintana S. Depression in Parkinson's disease: clinical correlates and outcome. Parkinson Relat Disord 2003; 10:23-8.

26. Schrag A, Jahanshahai M, Quinn NP. What contributes to depression in Parkinson's disease? Psychol Med 2001; 31:65-73.

27. Tandberg E, Larsen JP, Aarsland D, Laake K, Cummings $\mathrm{JL}$. Risk factors for depression in Parkinson disease. Arch Neurol 1997; 54:625-30.

28. Cubo E, Bernard B, Leurgans S, Raman R. Cognitive and motor function in patients with Parkinson's disease with and without depression. Clin Neuropharmacol 2000; 23:331-4.

29. Errea JM, Ara JR. Depression and Parkinson disease. Rev Neurol 1999; 28:694-8. 
30. Popovska A, Petrova V, Tanoska N, Vaskov T. Depressive symptoms in patients with parkinson's disease. J Neurol Sci Suppl 1997; 150: s113-4.

31. Starkstein SE, Prezioni T, Berthier M, Bolduc P, Mayberg $\mathrm{H}$, Robinson R. Depression and Cognitive Impairment in Parkinson's Disease. Brain 1989; 112:1141-53.

32. Choi C, Sohn YH, Lee JH, Kim J. The effect of long-term levodopa therapy on depression level in de novo patients with Parkinson's disease. J Neurol Sci 2000; 172:12-6.

33. Mayeux R. The serotonin hypotesis for depression in Parkinson's disease. Adv Neurol 1990; 53:163-6.

34. Sano M, Stern Y, Cote L, Williams JB, Mayeux R. Depression in Parkinson's disease: a biochemical model . J Neuropsychiatry Clin Neurosc 1990; 2:88-92.

35. Hoogendijk WJG, Purba JS, Hofman MA, Vos RAI, Jansen ENH, Swaab DF. Depression in Parkinson's Disease Is Not Accompanied by More Corticotropin-Releasing Hormone Expressing Neurons in the Hypothalamic Paraventricular Nucleus. Biol Psychiatry 1997; 43:913-7.

36. American Psychiatric Association. Task Force on Nomenclature and Statistics. Diagnostic and Statistical Manual of Mental Disorders. 3th ed. USA: American Psychiatric Association, 1980.

37. American Psychiatric Association. Task Force on Nomenclature and Statistics. Diagnostic and Statistical Manual of Mental Disorders. 4th ed. USA: American Psychiatric Association, 1994.

38. Shulman LM, Taback RL, Rabinstein AA, Weiner WJ. Non-recognition of depression and other non-motor symptoms in Parkinson's disease. Parkinson Relat Disord 2002; 8:193-7

39. Silberman CD, Marinho V, Laks J, Engelhardt E. Demência e doença de Parkinson. Revisão de publicações brasileiras de 1980 a 2000. Rev bras Neurol 2002; 38:22-30.

40. Liu CY, Wang SJ, Fuh JL, Yang YY, Liu HC. The correlation of depression with functional activity in Parkinson's disease. J Neurol 1997; 244:493-8.

41. Serrano-Dueñas M. Parkinson's disease, side affected and depression. Rev Neurol 2000; 31:1109-12.

\section{RESUMO}

Objetivos: Esta revisão tem dois objetivos. 1. Examinar o impacto da depressão sobre a cognição na Doença de Parkinson (DP). 2. Examinar o papel da depressão como fator de risco tanto para DP como para transtorno cognitivo na $D P$.

Metodologia: Revisão na literatura internacional, Medline, de artigos clínicos seccionais, prospectivos e de caso controle, avaliando a função cognitiva de parkinsonianos com e sem depressão, entre 1967 e 2003. Palavras-chave Doença de Parkinson, Depressão e cognição.

Resultados: Os trabalhos sobre o impacto da depressão na cognição de parkinsonianos apresentam afirmações divergentes. Cinco artigos concluem que há impacto e quatro não confirmam esses dados.

Pode-se afirmar que a depressão é um fator de risco para DP, assim como a DP é um fator de risco para depressão. No entanto, nenhuma definição fol possível no que se refere à depressão como fator de risco para transtornos cognitivos em parkinsonianos. Observa-se que os dados sobre a prevalência de depressão e déficit cognitivo na DP são inconclusivos, com grande margem percentual entre os autores.
A depressão em parkinsonianos está associada com avanço da gravidade da DP, estágio avançado de Hoehn e Yahr, alta pontuação na Unified Parkinson's Disease Rating Scale (UPDRS), ocorrência de quedas, baixa pontuação no Mini Exame do Estado Mental (MEEM) e na Escala Schwab e England, déficit cognitivo, bradicinesia axial, alterações na marcha e no balanço, idade mais avançada, sexo feminino e presença de alteração do pensamento.

Conclusão: A interação entre depressão e DP é complexa e bidirecional. A depressão é um fator de risco para DP, assim como DP é um fator de risco para depressão. É possível se traçar um perfil mais homogêneo do paciente deprimido com DP que evolui com transtorno cognitivo, mas não foi possível definir a depressão como um fator de risco para transtornos cognitivos na DP. Estudos que utilizem critérios diagnósticos definidos e com amostras representativas da população podem trazer esclarecimento sobre o assunto.

Descritores: Doença de Parkinson, depressão e cognição.

\section{ABSTRACT}

Objective: The objectives are twofold: 1- To review the impact of depression on cognition of patients with Parkinson's disease (DP) 2- To assess whether depression is a risk factor for $P D$ and cognitive deficit in $P D$.

Methodology: The bibliographical review considered the 1967-2003 period (Medline), using the keywords Parkinson's disease, depression and cognition. Only clinical cross-sectional, prospective and case control studies were included.

Results: Five articles showed a positive conclusion as to the impact of depression on cognition in $P D$, whereas four showed the opposite.

Depression is a risk factor for $P D$ as $P D$ is a risk factor for depression. On the other hand, it was impossible to draw a conclusion about depression as a risk factor for cognitive disorders in patients with $P D$.

Depression in patients with $P D$ is associated with advancing disease severity, high Hoehn and Yahr, high Unified Parkinson's Disease Rating Scale (UPDRS), falls, low Mini-Mental State Examination (MMSE) and Schwab and England scores, impaired cognitive functions, axial bradykinesia, gait and balance impairment, advanced age, female gender and thought disorders.

Conclusion: The interaction between depression and $P D$ is complex and bi-directional. Therefore, depression is a risk factor for $P D$ as $P D$ is a risk factor for depression. There is a homogeneous profile of depression patients with DP at risk to develop cognitive disorders. On the other hand, it is not possible to establish depression as a risk factor for cognitive disorders in PD. Further studies with 
representative samples and defined diagnosis criteria are needed to provide more information on this subject.

Keywords: Parkinson's disease, depression and cognition.

Title: A review of depression as a risk factor in Parkinson's disease and the impact on cognition

\section{RESUMEN}

Objetivos: Este trabajo tiene dos objetivos: 1) Examinar el impacto de la depresión sobre la cognición en el Mal de Parkinson (MP). 2) Examinar el papel de la depresión como factor de riesgo tanto para el MP como para trastorno cognoscitivo en el MP.

Metodología: Revisión de la literatura internacional (Medline), de artículos clínicos, seccionales, prospectivos y de caso control evaluando la función cognoscitiva de Parkinsonianos con y sin depresión, entre 1967 y 2003. Palabras clave: Mal de Parkinson, Depresión y Cognición.

Resultados: Los trabajos sobre el impacto de la depresión en la cognición de parkinsonianos presentan afirmaciones divergentes. Cinco artículos concluyen que hay impacto e cuatro no confirman esos datos.

Puede afirmarse que la depresión es un factor de riesgos para MP así como el MP es un factor de riesgo para la depresión. No en tanto, ninguna definición fue posible en lo que se refiere a la depresión como factor de riesgo para trastornos cognoscitivos en parkinsonianos. Se observa que los datos sobre a prevalencia de la depresión y déficit cognoscitivo en el MP son inconclusivos con grande margen porcen- tual entre los autores.

La depresión en parkinsonianos está asociada con el avance de la gravedad del MP, estado avanzado de Hoehn y Yahr, puntuación alta en la Unified Parkinson's Disease Rating Scale (UPDRS) ocurrencia de caídas, baja puntuación en el Mini Examen del Estado Mental (MEEM) y en la escala de Schwab y England, déficit cognoscitivo, bradykinesia axial, alteraciones en el caminar y en el balance, edad más avanzada, sexo femenino y presencia de alteración del pensamiento.

Conclusión: La interacción entre la depresión y MP es compleja y bi-direccional. La depresión es un factor de riesgo para MP así como MP es un factor de riesgo para la depresión. Es imposible trazar un perfil mas homogéneo del paciente deprimido con MP que desenvuelve trastorno cognoscitivo pero no fue posible definir la depresión como un factor de riesgo para trastornos cognoscitivos en el MP. Estudios que utilicen criterios diagnósticos definidos y con muestras representativas de la población puede traer un esclarecimiento sobre el asunto.

Palabras-claves: Mal de Parkinson, depresión y cognición.

Título: Revisión sobre la depressión como factor de riesgo en el Mal de Parkinson e impacto sobre la cognición

Endereço para correspondência: Jerson Laks

Av. Nossa Senhora de Copacabana, 749/802 22050-00 - Rio de Janeiro - RJ

E-mail: jlaks@centroin.com.br

Copyright $\odot$ Revista de Psiquiatria do Rio Grande do Sul - SPRS 